\title{
A quantum molecular similarity analysis of changes in molecular electron density caused by basis set flotation and electric field application
}

\author{
Sílvia Simon and Miquel Duran ${ }^{\text {a) }}$ \\ Department of Chemistry and Institute of Computational Chemistry, University of Girona, 17071 Girona, \\ Catalonia, Spain
}

(Received 16 December 1996; accepted 23 April 1997)

\begin{abstract}
Quantum molecular similarity (QMS) techniques are used to assess the response of the electron density of various small molecules to application of a static, uniform electric field. Likewise, QMS is used to analyze the changes in electron density generated by the process of floating a basis set. The results obtained show an interrelation between the floating process, the optimum geometry, and the presence of an external field. Cases involving the Le Chatelier principle are discussed, and an insight on the changes of bond critical point properties, self-similarity values and density differences is performed. (C) 1997 American Institute of Physics. [S0021-9606(97)01129-X]
\end{abstract}

\section{INTRODUCTION}

The theory of molecular structure proposed by Bader and co-workers ${ }^{1,2}$ uses the topological properties of the electron density to define certain properties of atoms in molecules. The charge density, $\rho(\mathbf{r})$, is a physical quantity which has a definite value at each point in space. Its topological properties are characterized in terms of the number and kind of its critical points. Such points satisfy the condition

$$
\nabla \rho(r)=0,
$$

where the first derivatives of $\rho(\mathbf{r})$ vanish and determine the position of extreme in the charge density, namely maxima, minima, or saddle points.

The bonding interaction between two atoms leads to formation of a critical point on the charge density surface. Critical points on the bond paths (the paths between bonded atoms along which the charge density is maximum with respect to a lateral displacement) are called bond critical points, $\mathbf{r}_{c}$.

The electron density, upon which this theory is based, is uniquely defined in terms of the wave function for any $\mathrm{N}$-electron system, and as a one-electron property it is correct to first order in the Hartree-Fock theory. This electron density changes when the system is perturbed, for instance when an external uniform electric field is applied. Such a change (relaxation of the electronic cloud) is better described, when using midsized basis sets, if floating functions are used. ${ }^{3-5}$ These basis functions are characterized by not being centered on any atomic nucleus, and by their position in space being optimized. One of their properties is that they mimic the behavior of polarization functions. Furthermore, they allow for better determination of electric properties by $a b$ initio methods. ${ }^{6}$

Since evaluation of electron properties relies many times on calculations involving application of an electric field, understanding the relationship between the floating process, the field strength, and the density topological properties is of great importance. The goal of the present research is to ana-

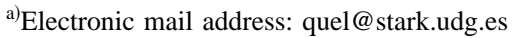

lyze the response of the electron density $\rho(\mathbf{r})$ to the floating process and to electric field application by means of quantum molecular similarity techniques. In particular, this work investigates the dependence of the position of the bond critical point, as well as the changes in $\rho\left(\mathbf{r}_{c}\right)$ and $\nabla^{2} \rho\left(\mathbf{r}_{c}\right)$, upon perturbation of the molecular system by a static, uniform electric field or by floating a basis set. Another purpose of this paper is to assess the changes in self-similarity with the variation of field strength or with floating. This study will deal with the systems $\mathrm{HF}, \mathrm{H}_{2} \mathrm{O}, \mathrm{NH}_{3}, \mathrm{CH}_{4}$, and $\mathrm{H}_{2}$, although special attention will be paid to HF, because the behavior of its electron density is more easily analyzable and thus the theoretical insight can be deeper

\section{METHODOLOGY}

This paper makes use of molecular similarity techniques, which is a novel, nice way to assess the changes in the electron densities (or differences between molecules). During the last years, quantum molecular similarity measures (QMSM) have been shown to be an efficient tool to compare two different one-electron densities. ${ }^{7,8}$ Two molecules, $A$ and $B$, which are described by one-electron densities $\rho_{A}(\mathbf{r})$ and $\rho_{B}(\mathbf{r})$, respectively, can be compared using a QMSM which is defined as the integral

$$
Z_{A B}(\Phi)=\rho_{A}\left(\mathbf{r}_{1}\right) \Phi\left(\mathbf{r}_{1}, \mathbf{r}_{2}\right) \rho_{B}\left(\mathbf{r}_{2}\right) d \mathbf{r}_{1} d \mathbf{r}_{2},
$$

where $\Phi\left(\mathbf{r}_{1}, \mathbf{r}_{2}\right)$ is a positive definite operator which depends on the electron coordinates. ${ }^{9-11}$ When this operator is a dirac delta function $\delta\left(\mathbf{r}_{1}-\mathbf{r}_{2}\right)$, it becomes an overlaplike QMSM, while when $\Phi$ is the Coulomb operator $\left(\mathbf{r}_{1}-\mathbf{r}_{2}\right)^{-1}, Z_{A B}$ becomes the so-called Coulomb-like QMSM.

The quantum molecular self-similarity measure of a particular molecule, $Z_{A A}$, can be obtained from the diagonal similarity matrix elements. From a quantum-mechanical point of view, the overlaplike self-similarity measure can be considered as the expectation value of the density operator, and therefore as an observable $;{ }^{12}$ furthermore, it can also be considered as an indicator of the concentration of charge. Likewise, the Coulomb-like self-similarity measure is the expectation value corresponding to the electronic term of the 
TABLE I. Bond critical points $\left(\mathbf{r}_{c}\right)$ of hydrogen fluoride at different field strengths $(\mathbf{F})$, and derivative of the bond critical point position with respect to electric field strength (in a.u.). No floating function $[\operatorname{HF}(G)]$, function-only optimization $[\mathrm{HF}(F)]$ and floating functions calculations $[\mathrm{HF}(F G)]$.

\begin{tabular}{rrrr}
\hline \hline Field strength & $\mathrm{HF}(G)$ & $\mathrm{HF}(F)$ & $\mathrm{HF}(F G)$ \\
\hline-0.04 & 0.3719 & 0.3861 & 0.3825 \\
-0.03 & 0.3647 & 0.3789 & 0.3755 \\
-0.02 & 0.3575 & 0.3718 & 0.3685 \\
-0.01 & 0.3503 & 0.3648 & 0.3616 \\
0.00 & 0.3431 & 0.3577 & 0.3547 \\
0.01 & 0.3360 & 0.3508 & 0.3479 \\
0.02 & 0.3288 & 0.3438 & 0.3410 \\
0.03 & 0.3216 & 0.3369 & 0.3342 \\
0.04 & 0.3145 & 0.3299 & 0.3273 \\
$d\left(\mathbf{r}_{c}\right) / \mathbf{F}$ & -0.7177 & -0.7013 & -0.6890 \\
\hline \hline
\end{tabular}

molecular electrostatic potential and can be considered as an indicator of the amount of repulsion between electron pairs.

Different indexes can be defined from the matrix elements of the optimal (maximum with respect to mutual orientation) similarity $Z_{A B}$. Two classical, well-characterized indexes are the Carbó index, ${ }^{7,8} I_{A B}$, which represents the generalized cosines between the $\rho_{A}$ and $\rho_{B}$ vectors, as given by

$$
I_{A B}=\frac{Z_{A B}}{\sqrt{Z_{A A} Z_{B B}}}
$$

and the euclidean distance $D_{A B},{ }^{7,8}$ which is defined by

$$
D_{A B}=\sqrt{Z_{A A}+Z_{B B}-2 Z_{A B}} \text {. }
$$

The GAUSSIAN $92^{13}$ series of programs was used for all computations, which were made at Hartree-Fock level of theory. Floating function calculations were carried out using ghost atoms as function centers. The different floating schemes used in the research are described in the text when required. The Huzinaga-Dunning DZ basis set $^{14}$ was used everywhere. Overlaplike QMSM and Coulomb-like QMSM were calculated starting from GAUSSIAN92 electron densities using the MESSEM ${ }^{15}$ program. Bader topological properties of the charge density were determined using the ELECTRA ${ }^{16}$ series of programs developed in our research group.

\section{RESULTS AND DISCUSSION}

This section is organized as follows: First, we examine the change in properties of the bond critical point $\left(\mathbf{r}_{c}\right)$ in the $\mathrm{HF}$ molecule when a uniform electric field is applied as well as its change in other hydrides; second, we perform an analysis of the quantum molecular self-similarity measures obtained in a series of test molecules; and third, density difference maps in HF are analyzed.

\section{Analysis of the bond critical point of HF and other hydrides}

In order to calculate the electric-field dependence of the bond critical point, single-point calculations (no geometry reoptimization) were performed on different hydrides perturbed by an electric field having different strengths, in a direction parallel to the chemical bond $\mathrm{A}-\mathrm{H}$. Tables I and Tables II gather the bond critical point positions $\left(\mathbf{r}_{c}\right)$, the electron density values $\left[\rho\left(\mathbf{r}_{c}\right)\right]$ and the Laplacian $\left[\nabla^{2} \rho\left(\mathbf{r}_{c}\right)\right]$ values found for the hydrogen fluoride molecule. Three different floating situations are considered: first, a nonfloating (fixed) basis set $[\mathrm{HF}(G)]$, second, function flotation at non-floating-optimized geometry $[\operatorname{HF}(F)]$, and finally, simultaneous optimization of the nuclei position (i.e., geometry) and basis function position (i.e., floating) $[\operatorname{HF}(F G)]$.

TABLE II. Electron-density $\left[\rho\left(\ddot{U} \mathbf{r}_{c}\right)\right]$ and its Laplacians $\left[\nabla^{2}\left(\mathbf{r}_{c}\right)\right]$ for hydrogen fluoride for different field strengths (in a.u.). No floating $[\mathrm{HF}(G)]$, function-only optimization $[\operatorname{HF}(F)]$, and floating plus geometry

\begin{tabular}{|c|c|c|c|c|c|c|}
\hline \multirow{2}{*}{$\begin{array}{l}\text { Field } \\
\text { strength }\end{array}$} & \multicolumn{2}{|c|}{$\mathrm{HF}(G)$} & \multicolumn{2}{|c|}{$\operatorname{HF}(F)$} & \multicolumn{2}{|c|}{$\mathrm{HF}(F G)$} \\
\hline & $\rho\left(\mathbf{r}_{c}\right)$ & $\nabla^{2}\left(\mathbf{r}_{c}\right)$ & $\rho\left(\mathbf{r}_{c}\right)$ & $\nabla^{2}\left(\mathbf{r}_{c}\right)$ & $\rho\left(\mathbf{r}_{c}\right)$ & $\nabla^{2}\left(\mathbf{r}_{c}\right)$ \\
\hline-0.04 & 0.3623 & -1.6574 & 0.3618 & -1.5956 & 0.3672 & -1.6324 \\
\hline-0.03 & 0.3597 & -1.6886 & 0.3594 & -1.6197 & 0.3649 & -1.6583 \\
\hline-0.02 & 0.3568 & -1.7223 & 0.3568 & -1.6452 & 0.3623 & -1.6857 \\
\hline-0.01 & 0.3537 & -1.7592 & 0.3540 & -1.6726 & 0.3594 & -1.7152 \\
\hline 0.0 & 0.3503 & -1.8001 & 0.3510 & -1.7025 & 0.3564 & -1.7476 \\
\hline 0.01 & 0.3468 & -1.8453 & 0.3477 & -1.7356 & 0.3532 & -1.7832 \\
\hline 0.02 & 0.3431 & -1.8959 & 0.3444 & -1.7724 & 0.3498 & -1.8228 \\
\hline 0.03 & 0.3392 & -1.9522 & 0.3408 & -1.8135 & 0.3462 & -1.8669 \\
\hline 0.04 & 0.3351 & -2.0151 & 0.3371 & -1.8596 & 0.3425 & -1.9160 \\
\hline
\end{tabular}
optimization calculations $[\mathrm{HF}(F G)]$. 


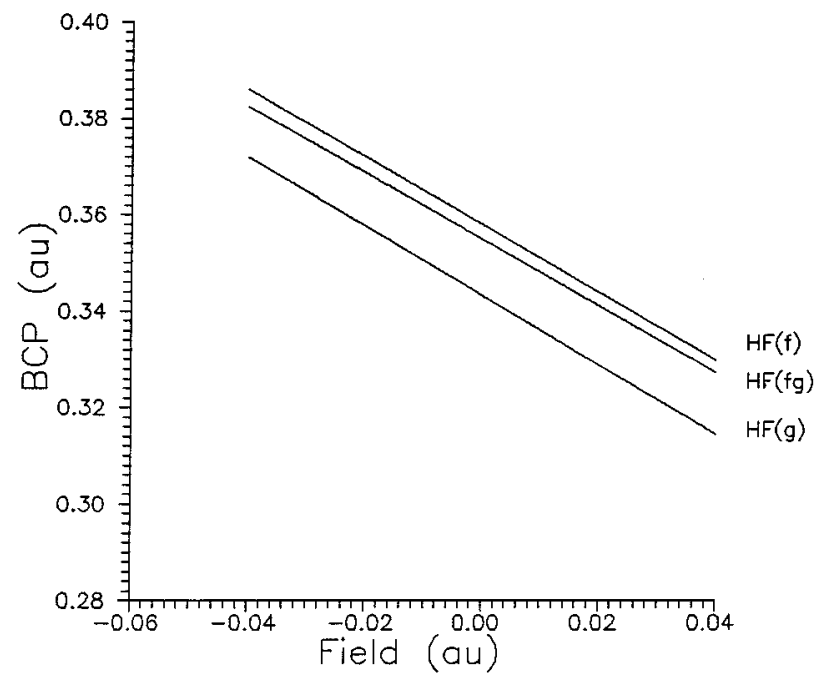

FIG. 1. Bond critical point position (a.u.) vs electric field strength (a.u.).

The optimized bond lengths turn out to be $0.9196 \AA$ ( $F$ and $G)$ and $0.9146 \AA(F G)$, which can be compared to the experimental value of $0.917 \AA \AA^{17}$

When a uniform electric field is applied and no geometry optimization is allowed, the position of the bond critical point $\left(\mathbf{r}_{c}\right)$ exhibits a linear behavior, as shown in Fig. 1. Fixed-function and floating-function calculation have more or less the same slope, the bond critical point position at zero field are the only to change. Figure 2 plots the relationship between charge density at the bond critical point and field strength, whereas Fig. 3 depicts the Laplacian values at the bond critical point vs different electric field strengths. These figures show that densities at the bond critical point and Laplacian values $\left[\rho\left(\mathbf{r}_{c}\right), \nabla^{2} \rho\left(\mathbf{r}_{c}\right)\right]$ exhibit an opposite behavior when strong positive electric field are applied: While the charge density decreases due to the fact that $\mathbf{r}_{c}$ also decreases

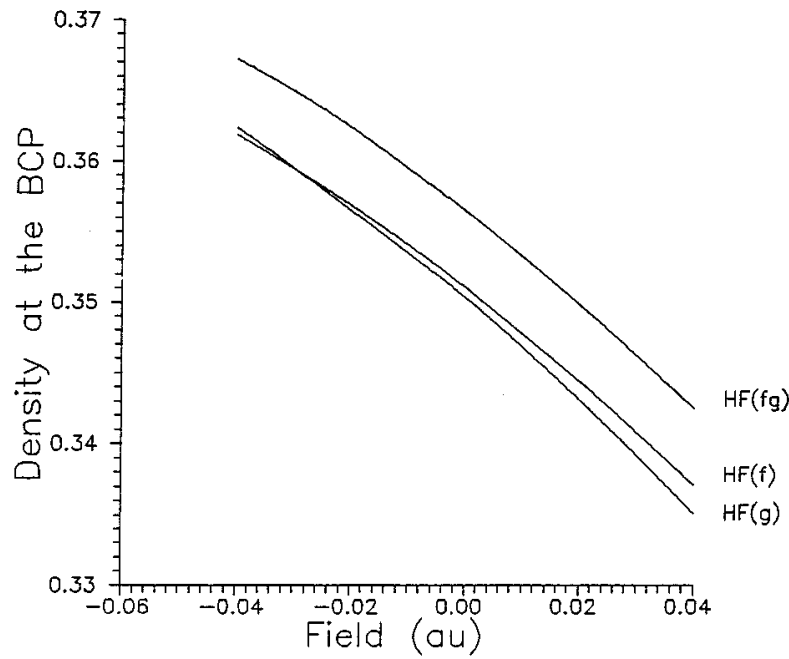

FIG. 2. Charge density (a.u.) at the bond critical point vs electric field strength (a.u.).

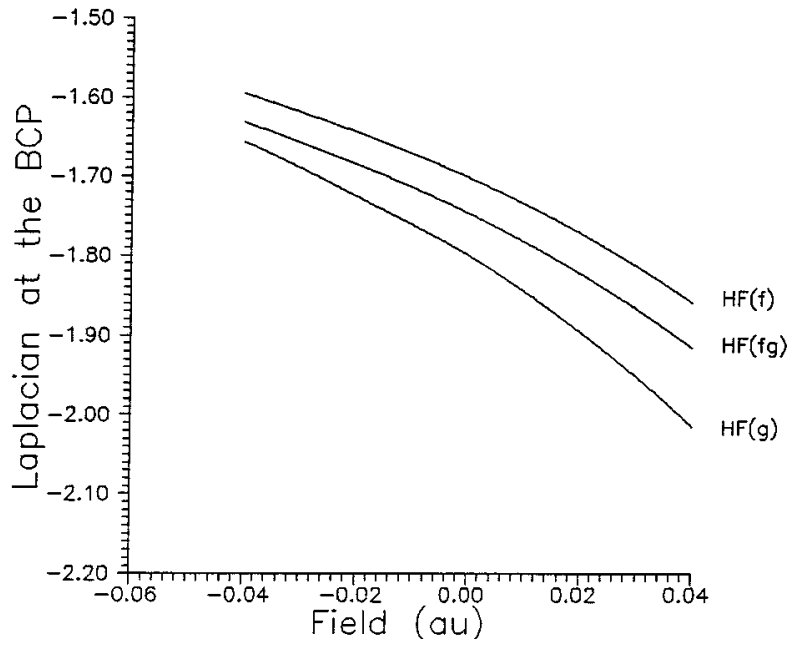

FIG. 3. Laplacian (a.u.) vs electric field strength (a.u.).

(i.e., it approaches the $\mathrm{H}$ atom) Laplacian values become more negative, which means there is an increase in the charge concentration.

Comparing fixed-function $(G)$ with function-only $(F)$ and simultaneous function and nuclei optimization calculations $(F G)$, one can observe an example of the quantum chemical Le Chatelier principle. ${ }^{18}$ When functions are allowed to move independently from nuclei, $\mathbf{r}_{c}, \rho\left(\mathbf{r}_{c}\right)$, $\nabla^{2} \rho\left(\mathbf{r}_{c}\right)$, and $d\left(\mathbf{r}_{c}\right) / d \mathbf{F}$ change in one direction, while when one reoptimizes the geometry using a floating basis set, the values change in the opposite direction, and are able to readjust thus fulfilling the quantum chemical Le Chatelier principle.

For other hydrides, $(A H)$, Table III collects the partial derivative of the bond critical point position with respect to electric field strength (the slope of the straight line), electronegativities, bond length, the distance between the bond critical point and the hydrogen atom, and the electron density at this point. Furthermore, an interesting logarithmic relationship between the type of heavy atom and the position of the bond critical point is found. Fig. 4 plots the electronegativity $\chi_{A}$ of the heavy atom vs $d\left(\mathbf{r}_{c}\right) / d \mathbf{F}$, the following formula being obtained:

$$
\frac{d \mathbf{r}_{c}}{d \mathbf{F}}=18.707\left(\chi_{A}^{-2.36}\right) .
$$

TABLE III. Derivative of bond critical point positions of HA systems with respect to electric field strength (a.u.), along with electronegativity $\left(\chi_{\mathrm{A}}\right)$, bond length $\left(\mathbf{r}_{\mathrm{AH}} \AA\right)$, bond critical point distance from $\mathrm{H}\left(\mathbf{r}_{\mathrm{H}} \AA\right)$ and charge density at the bond critical point $\left[\rho\left(\mathbf{r}_{c}\right)\right]$ in a.u.

\begin{tabular}{lccccc}
\hline \hline & $d\left(\mathbf{r}_{c}\right) / d \mathbf{F}$ & $\chi_{\mathrm{A}}$ & $\mathbf{r}_{\mathrm{AH}}$ & $\mathbf{r}_{\mathrm{H}}$ & $\rho\left(\mathbf{r}_{c}\right)$ \\
\hline$-\mathrm{F}$ & -0.718 & 4.0 & 0.9196 & 0.181 & 0.3503 \\
$-\mathrm{OH}$ & -0.892 & 3.64 & 0.9513 & 0.211 & 0.3482 \\
$-\mathrm{NH}_{2}$ & -1.290 & 3.10 & 0.9944 & 0.262 & 0.3257 \\
$-\mathrm{Cl}$ & -1.922 & 3.05 & 1.2952 & 0.402 & 0.2072 \\
$-\mathrm{CH}_{3}$ & -2.040 & 2.56 & 1.0834 & 0.418 & 0.2581 \\
\hline \hline
\end{tabular}




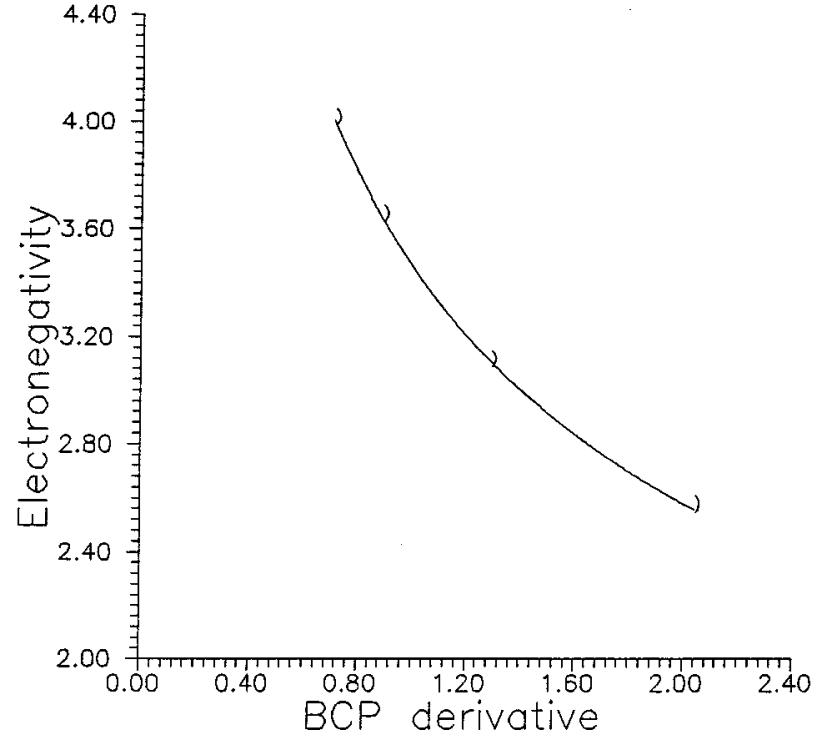

FIG. 4. Derivative of bond critical point (a.u.) vs electric field strength (a.u.).

Data collected in Table III show clearly that the derivative of the bond critical point position, with respect to the electric field strength, increases with the electronegativity of the heavy atom and with the decrease in $\rho\left(\mathbf{r}_{c}\right)$. This may be due to the decrease in charge concentration at the bond critical point, so $\mathbf{r}_{c}$ has more freedom to move. Boyd et al. ${ }^{19}$ already showed that $\rho\left(\mathbf{r}_{c}\right)$ increases monotonically within each period of the periodic table as the atomic number increases. It can be thus understood that $d\left(\mathbf{r}_{c}\right) / d \mathbf{F}$ decreases along the period. Moreover, bond critical point distance form $\mathrm{H}$ atom $\left(\mathbf{r}_{\mathrm{H}}\right)$ decreases upon increase of electronegativity of $A$ because of the greater tendency of the electron cloud to migrate to $A$, thus causing $r_{\mathrm{H}}$ to be smaller.

\section{QMSM of small molecules}

So far, we have dealt with an important, yet local property. In this part of our research, we will proceed to a more global analysis of the modifications in $\rho\left(\mathbf{r}_{c}\right)$ caused by an externally applied field.

In Table IV we present the exact (i.e., Hartree-Fock densities not fitted to a series of auxiliary functions) overlaplike and Coulomb-like QMS measures for the hydrogen molecule and a series of four isoelectronic hydrides containing 10 electrons (the DZ basis set has been used throughout). For these systems, three different situations have been considered: $G$ stands for plain fixed-basis geometry optimization; $F$ stands for function floating (nuclei fixed in space) using the previously optimized geometry; and finally, $F G$ stands for simultaneous function and geometry optimization. Furthermore, similar calculations have been performed with each molecule being perturbed by a static, uniform electric field having a strength of 0.04 a.u., as depicted in Fig. 5.

Table IV can be analyzed in several ways. First of all, the value for overlap- and Coulomb- $Z_{A A}$ is indeed much smaller for $\mathrm{H}_{2}$ than for the other molecules, because of the
TABLE IV. Exact $a b$ initio Hartree-Fock overlap and Coulomb (in italic) quantum molecular self-similarities for different molecules computed with the DZ basis set (in a.u.).

\begin{tabular}{lccccc}
\hline \hline \multirow{2}{*}{$G$} & $\mathrm{H}_{2}$ & $\mathrm{CH}_{4}$ & $\mathrm{NH}_{3}$ & $\mathrm{H}_{2} \mathrm{O}$ & $\mathrm{HF}$ \\
\hline \multirow{2}{*}{$F$} & 0.171 & 31.888 & 52.515 & 81.401 & 119.934 \\
& 2.636 & 65.406 & 78.578 & 93.592 & 111.560 \\
& 0.175 & 31.877 & 52.493 & 81.372 & 119.911 \\
$F G$ & 2.661 & 65.466 & 78.628 & 93.647 & 111.612 \\
& 0.171 & 31.876 & 52.509 & 81.379 & 119.915 \\
$F 4$ & 2.636 & 65.462 & 78.600 & 93.692 & 111.655 \\
& 0.168 & 31.883 & 52.547 & 81.342 & 119.866 \\
$F 4$ & 2.621 & 65.279 & 78.315 & 93.711 & 111.641 \\
& 0.174 & 31.880 & 52.471 & 81.306 & 119.857 \\
\multirow{3}{*}{$F G 4$} & 2.656 & 65.421 & 78.681 & 93.821 & 111.802 \\
& 0.173 & 31.871 & 52.531 & 81.327 & 119.849 \\
& 2.646 & 65.335 & 78.397 & 93.741 & 111.731 \\
\hline \hline \multirow{3}{*}{$F$} & & & & &
\end{tabular}

difference in the number of electrons. As to first-row hydrides, the values of $Z_{A A}$ increase with the atomic number of the heavy atom, because of the increase of electron charge concentration around its nucleus. ${ }^{20}$

Although the trend with both types of $Z_{A A}$ is the same, the difference between $\mathrm{HF}$ and $\mathrm{CH}_{4} Z_{A A}$ values depends on the operator being used: actually, overlap- and Coulomb$Z_{A A}$ values are quite similar for $\mathrm{HF}$, whereas they are quite different for $\mathrm{CH}_{4}$. The reason is found in the values of the two measures which stand for: Overlap QMSM measure the amount of charge concentration, whereas Coulomb QMSM measure the amount of electron-electron repulsion. Similar trends were already shown in an earlier paper by our Group, where first- and second-row hydrides and other molecules were studied. $^{20}$ In fact, the values for the $\mathrm{Ne}$ atom were
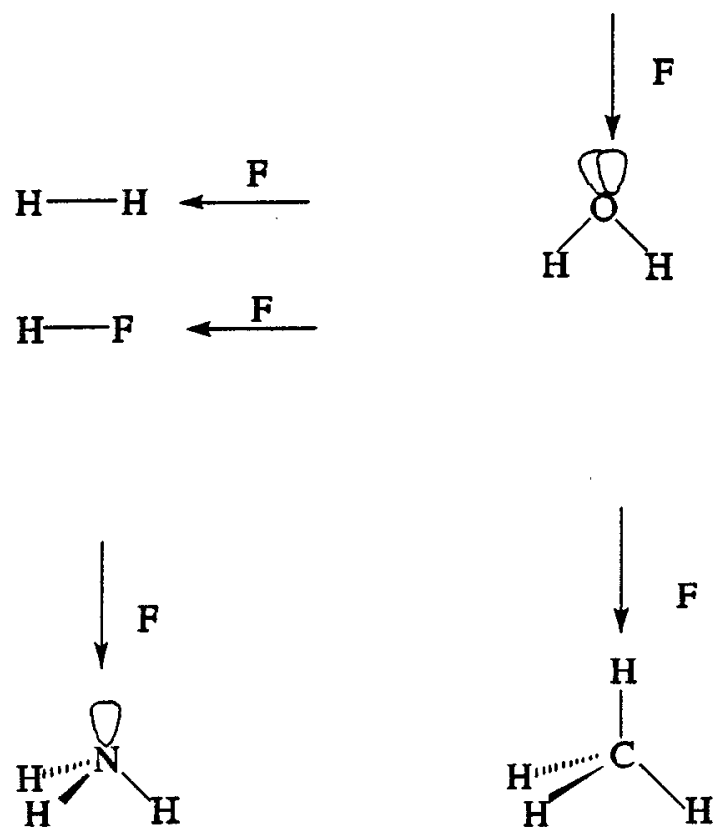

FIG. 5. Directions of the electric field in the different molecules studied. 
computed in that paper (170.127 for overlap $Z_{A A}$ and 132.172 for repulsion $Z_{A A}$ ), which are consistent with the values found in the present paper.

A consequence of the different behavior of overlap and Coulomb-based QMSM is that the effects of perturbations like the electric field, which polarizes the electronic cloud, or the floating process of the basis functions, are more reflected in the Coulomb-like measures. Let us consider first the fieldfree measures. Except for $\mathrm{H}_{2}$, overlap-based QMS measures show that floating the basis functions $(F)$ after fixed-basis geometry optimization $(G)$ decreases the $Z_{A A}$ values, because the electronic cloud becomes more diffuse, i.e., the electron charge becomes more disperse. However, further reoptimization $(F G)$ again increases the values of $Z_{A A}$, so as to approach the original $G$ (plain, fixed functions) values. In a sense, new forces appear on nuclei that tend to move them and therefore concentrate charge again.

On the contrary, Coulomb-type QMS values follow an opposite trend: They increase with flotation of the basis set $(F)$, whereas they decrease or stay constant with simultaneous floating and geometry optimization $(F G)$. The reason why the Coulomb-like $Z_{A A}$ follows a trend opposite to the overlaplike $Z_{A A}$ may be found in the fact that floating increases the repulsion between lone pairs of the heavy atoms.

These trends along the $G-F-G F$ series are preserved if a static, uniform electric field is applied $(G 4, F 4$, and $G F 4$ cases in Table IV), although the variations in $Z_{A A}$ are much larger, because of the concentration of charge (or change in repulsion) the field causes on all molecules. Furthermore, it is worth to note the different effect of field application on $Z_{A A}$ values: For overlaplike $Z_{A A}$, application of the field (G4) always decreases the QMSM values, except for $\mathrm{NH}_{3}$. On the contrary, for Coulomb-type QMSM, the field increases the $Z_{A A}$ values for $\mathrm{H}_{2} \mathrm{O}$ and $\mathrm{HF}$, while it decreases them for $\mathrm{H}_{2}, \mathrm{CH}_{4}$, and $\mathrm{NH}_{3}$. The reason of this apparrently erratic behavior can be understood by looking at two possibly opposite effects. For instance, let us compare $\mathrm{H}_{2} \mathrm{O}$ and $\mathrm{NH}_{3}$. For the former system, application of the field along the symmetry axis causes a dispertion of the electron cloud, which more than compensates the possible increase in charge concentration around the oxygen inner core electrons. At the same time, overall repulsion is increased because repulsion between the two lone pairs of oxygen is favored when electric filed is applied. For $\mathrm{NH}_{3}$, the second effect is reversed, because the repulsion decreases since the electrons on the $\mathrm{N}-\mathrm{H}$ bond become more separated in space; on the contrary, the dispersion of charge far than compensates for the increase in core contraction of the inner (core) electrons of nitrogen. Thus, the different behavior of these two molecules can be explained.

A further, deeper insight into the effect of floating and field application on $Z_{A A}$ values can be made from Figs. 6 and 7 , where the $Z_{A A}$ values for HF (calculated with the DZ basis set) are plotted. For this molecule, four different optimized geometries (minima in four different potential-energy surfaces) have been considered, depending on (a) floating being allowed or forbidden, and (b) an electric field being absent or applied. For each of the four geometries (actually bond

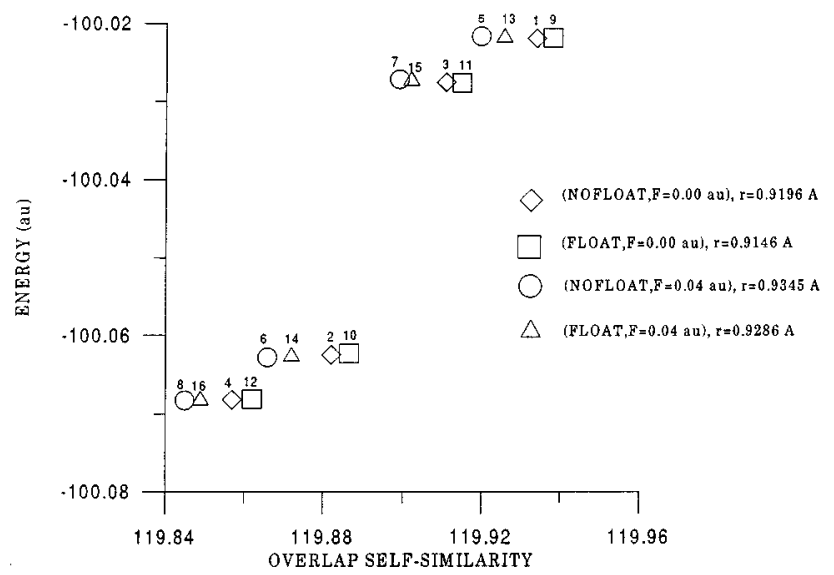

FIG. 6. Total energy (a.u.) vs overlap self-similarity.

length), floating and field application have been turned on and off, so 16 calculations have been performed. Thus, Figs. 6 and 7 plot the total-energy values overlap- or Coulomb$Z_{A A}$ values. Each of the 16 situations has been labeled and characterized in Table $\mathrm{V}$, which also reports dipole moments.

Comparison of Figs. 5 and 6 shows that overlap and repulsion self-similarity values follow the trend already hinted by Table IV: They behave oppositely. For each bond length, there are four situations, leading to four quite different energies, the lowest one always corresponding to field application and maximum freedom (i.e., simultaneous optimization of the basis function positions and nuclei positions). However, whereas overlap $Z_{A A}$ values decrease upon decrease in energy, the corresponding repulsion $Z_{A A}$ values increase when energies lower, in complete agreement with the values collected for HF in Table IV.

Regarding energies, it is clear that the effect of the field application (dependent on field strength, which is clearly very strong here) is much higher than that of floating. Thus, the series $\{1,5,9,13\}$ is close to series $\{3,7,11,15\}$, since they differ only in the floating process. Likewise, the other two

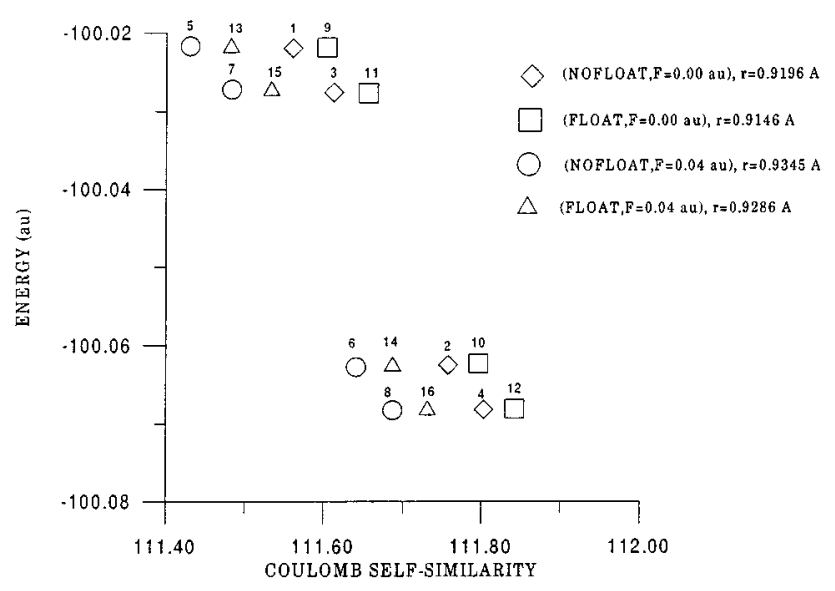

FIG. 7. Total energy (a.u.) vs Coulomb self-similarity. 
TABLE V. Bond length (§), total energy (a.u.), overlap and Coulomb quantum molecular self-similarity and dipole moments of hydrogen fluoride (a.u.) for different floating cases and field strength.

\begin{tabular}{rcccccc}
\hline \hline & $\mathbf{r}$ & Float,Field & $E$ & $Z_{A A}$ & $Z_{A A}\left(\mathbf{r}^{-1}\right)$ & $\mu$ \\
\hline 1 & $0.9196^{\mathrm{a}}$ & No,No & -100.021979 & 119.934 & 111.560 & -0.9374 \\
2 & & No,Yes & -100.062527 & 119.882 & 111.757 & -1.0858 \\
3 & & Yes,No & -100.027628 & 119.911 & 111.612 & -0.9412 \\
4 & & Yes,Yes & -100.068243 & 119.857 & 111.802 & -1.0856 \\
5 & $0.9345^{\mathrm{b}}$ & No,No & -100.021733 & 119.920 & 111.430 & -0.9461 \\
6 & & No,Yes & -100.062765 & 119.866 & 111.641 & -1.1012 \\
7 & & Yes,No & -100.027209 & 119.899 & 111.483 & -0.9495 \\
8 & & Yes,Yes & -100.068294 & 119.845 & 111.687 & -1.1005 \\
9 & $0.9146^{\mathrm{c}}$ & No,No & -100.021950 & 119.938 & 111.603 & -0.9346 \\
10 & & No,Yes & -100.062338 & 119.887 & 111.796 & -1.0808 \\
11 & & Yes,No & -100.027657 & 119.915 & 111.655 & -0.9384 \\
12 & & Yes,Yes & -100.068117 & 119.862 & 111.842 & -1.0807 \\
13 & $0.9286^{\text {d }}$ & No,No & -100.021889 & 119.925 & 111.482 & -0.9427 \\
14 & & No,Yes & -100.062728 & 119.872 & 111.687 & -1.0950 \\
15 & & Yes,No & -100.027434 & 119.902 & 111.533 & -0.9462 \\
16 & & Yes,Yes & -100.068332 & 119.849 & 111.731 & -1.0946 \\
\hline \hline
\end{tabular}

${ }^{\mathrm{a}}$ Nonfloating and zero-field geometry.

${ }^{\mathrm{b}}$ Nonfloating and field $=0.04$ a.u. geometry.

${ }^{c}$ Floating and zero-field geometry.

${ }^{\mathrm{d}}$ Floating and field $=0.04$ a.u. geometry.

lower-energy series differ in the floating process, although an electric field is applied there.

It is quite interesting to analyze the order of the series within each (floating, field) case: They always follow the same order. To make things easier, let us concentrate on the first series, corresponding to the field free nonfloating case: Points 5, 13, 1, and 9 correspond, respectively, to geometries optimized with four different schemes, as shown in Figs. 6 and 7, and in Table V. These four different geometries have four different bond lengths, as reported in Table V. Then, the relationship between interatomic distances and overlap $Z_{A A}$ emerges clearly: The longer the bond, the lower the overlap $Z_{A A}$, because the electron charge is more disperse. The same trend is shown in the repulsion $Z_{A A}$ : The longer the bond distance the lower repulsion in the charge density. The change in bond length may then explain the results found above for HF and other molecules.

For both Figs. 6 and 7, one can plot an imaginary path connecting the field-free, nonfloating system (labeled 1) with the field-perturbed, floating system (labeled 16). Obviously, 1 is the lowest energy among the highest series, whereas 16 is in turn the most stable system among all 16. Moreover, one can think of plotting other interesting paths involving selected geometry optimizations. For instance, a first process of floating followed by geometry reoptimization is traced by a line connecting points 1,3 , and 11 . Second, the process of applying an electric field followed by geometry reoptimization can be traced by a line connecting points 1,2 , and 6 . A third interesting example is the process of applying a field and floating, traced by a line connecting points 1,2 , and 4 . Indeed, many paths between 1 and 16 can be traced down.

The existence of a Le Chatelier-type behavior can be understood now easily: For overlap $Z_{A A}$, if floating is performed first, and another effect is taken, $Z_{A A}$ increases first

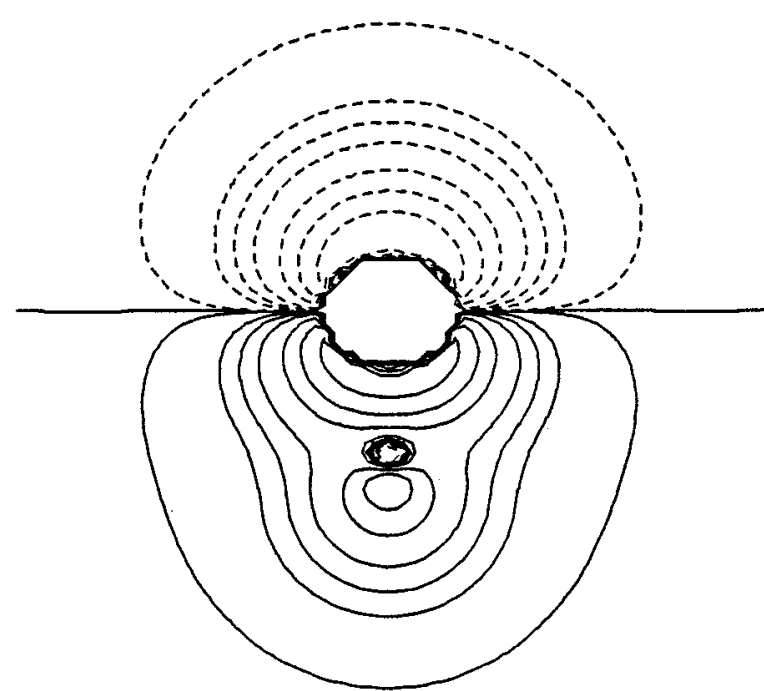

FIG. 8. Density difference map: $\rho$ (floating) $-\rho$ (nonfloating).

and decreases later. If the final point in the path is $11, Z_{A A}$ decreases first and then increases. In other cases, the changes depend on the case. For instance, case 2 above (field+reoptimization) decreases both times $Z_{A A}$, as case 3 (field + floating) does.

Since the effect of Coulomb- $Z_{A A}$ is exactly opposite to that of overlap- $Z_{A A}$, Le Chatelier-type behaviors are mutually exclusive for overlap and Coulomb- $Z_{A A}$. This analysis allows us to understand more clearly the behavior of these measures in HF and the other molecules in Table IV.

A final point worth analyzing concerns the values of the molecular dipole moment in Table V. Indeed, application of the field, which lowers the total energy, also increases the dipole moment. Floating the basis set also increases, although to a much lower extent, the dipole moment of HF. This increase can be understood by the separation of the basis functions of $\mathrm{F}$ and $\mathrm{H}$, so the internuclear distance is smaller than the distance between function centers, thus increasing the mean distance between changed centers.

\section{Electron density differences}

To better understand the changes in electron density caused by floating or field application, we have drawn the variations in electron density, at a constant geometry, caused by the floating process (Fig. 8) or by electric field application (Fig. 9).

The plot in Fig. 8 (H being under F) shows that floating, which pulls basic functions centers appart from nuclei, increases the electron density in the region around the $\mathrm{H}$ atom being opposite to the $\mathrm{F}$ atom. Floating the basis functions leads to a distribution of the density along the chemical bond. This is caused by the displacement of the functions in this direction. The contraction of the electronic cloud leads to a shorter electronic part of the dipole moment which results into an increase of the total dipole moment (Table V, points 1 and 3). 


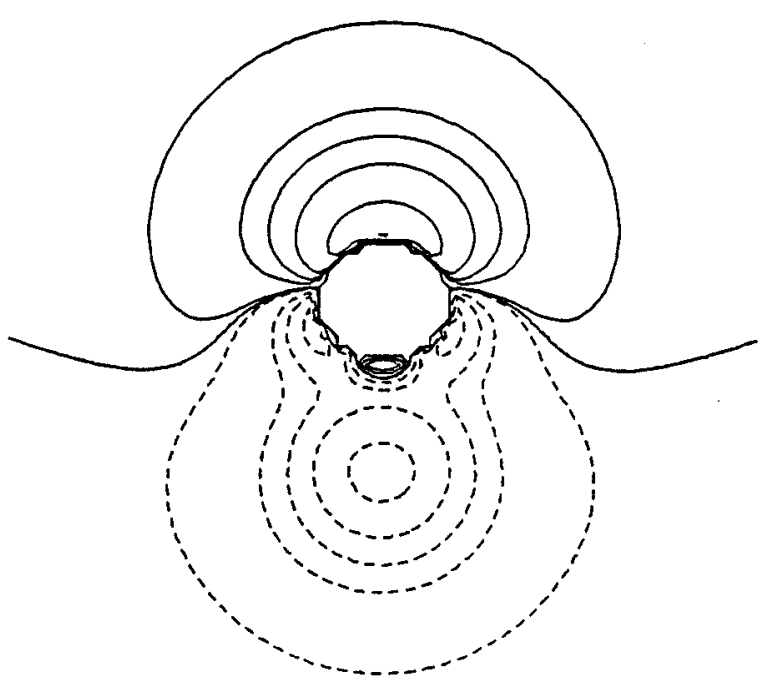

FIG. 9. Density difference map: $\rho$ (nonfloating, filed $=0.04$ a.u.) $-\rho$ (nonfloating, zero field).

On the contrary, application of the field (Fig. 9) increases the electron density around the $\mathrm{F}$ atom, while decreasing it around the $\mathrm{H}$ atom. This is due to the fact that the electric field is applied along the $\mathrm{H}-\mathrm{F}$ direction, so the density follows it. This can also be checked by the increase in dipole moment (Table V) caused by the field (points 1 and 2).

\section{CONCLUSIONS}

This work has shown the interrelation of the function floating process, the optimum geometry, and the application of an electric field to a particular molecule by carrying out a deep insight on the response of the electron density. In particular, good examples of the quantum Le Chatelier principle are found. Moreover, an assessment of the self-similarity overlap- and Coulomb-type QMSM values is made, the trend along an isoelectronic series being discussed. Finally, the change in bond critical point properties and self-similarity values are analyzed from density difference maps.

\section{ACKNOWLEDGMENTS}

The authors wish to thank Dr. J. Mestres for helpful comments. Financial help has been provided by the Spanish DGICYT through Project No. PB92-0333. S. Simon thanks also the Generalitat de Catalunya for a Ph.D. fellowship.

${ }^{1}$ R. F. W. Bader, Acc. Chem. Res. 18, 9 (1985).

${ }^{2}$ R. F. W. Bader, Atoms in Molecules: A Quantum Theory (Clarendon, Oxford, 1990).

${ }^{3}$ A. C. Hurley, The Molecular Orbital Interpretation of Bond-Length Changes Following Excitation and Ionization of Diatomic Molecules, edited by Lowdon and Pullman (Academic, New York, 1964).

${ }^{4}$ T. Helgaker and J. Almlöf, J. Chem. Phys. 89, 4889 (1988).

${ }^{5}$ K. Hirao and K. Mogi, J. Comput. Chem. 13, 457 (1992).

${ }^{6}$ S. Simon and M. Duran (unpublished).

${ }^{7}$ R. Carbó, M. Arnau, and L. Leyda, Int. J. Quantum Chem. 17, 1185 (1980).

${ }^{8}$ R. Carbó and B. Calabuig, Int. J. Quantum Chem. 42, 1681 (1992).

${ }^{9}$ R. Carbó, B. Calabuig, L. Vera, and E. Besalú, Adv. Quantum Chem. 25, 253 (1994).

${ }^{10}$ E. Besalú, R, Carbó, J. Mestres, and M. Solà, Molecular Similarity, Topics in Current Chemistry Series (Springer, Berlin, 1995), Vol. 173, pp. 31-62.

${ }^{11}$ R. Carbó and E. Besalú, Proceedings of the First Girona Seminar on Molecular Similarity (Kluwer, Dordrecht, 1995).

${ }^{12}$ R. Carbó and Ll. Domingo, Int. J. Quantum Chem. 32, 517 (1987).

${ }^{13}$ GaUssian 92, M. J. Frisch, G. W. Trucks, M. Head-Gordon, P. M. W. Gill, M. W. Wong, J. B. Foresman, R. G. Johnson, H. B. Schlegel, M. A. Robb, E. S. Replogle, R. Gomperts, J. L. Andrés, K. Raghavachari, J. S. Binkley, C. Gonzalez, R. L. Martin, D. J. Fox, D. J. Defrees, J. Baker, J. J. P. Stewart, and J. A. Pople, Gaussian, Inc., Pittsburgh, PA, 1992.

${ }^{14}$ T. H. Dunning, J. Chem. Phys. 53, 2823 (1970).

${ }^{15}$ J. Mestres, M. Solà, E. Besalú, M. Duran and R. Carbó, MEssem (Girona, CAT, 1993).

${ }^{16}$ J. Mestres, ELECTRA (Girona, CAT, 1994).

${ }^{17}$ Y. Yamaguchi, M. Frisch, J. Gaw, J. S. Binkley, and H. F. Shaeffer III, J. Chem. Phys. 84, 2262 (1986).

${ }^{18}$ P. G. Mezey, Int. J. Quantum Chem. 25, 853 (1984).

${ }^{19}$ R. J. Boyd and E. Edgecombe, J. Am. Chem. Soc. 110, 4182 (1988).

${ }^{20}$ M. Solà, J. Mestres, J. M. Oliva, M. Duran, and R. Carbó, Int. J. Quantum Chem. 58, 361 (1996). 